\title{
ATOMIC DATA
}

\section{DIVISION XII / COMMISSION 14 / WORKING GROUP ATOMIC DATA}

CHAIR
VICE-CHAIR

\author{
Gillian Nave \\ Glenn M. Wahlgren, Jeffrey R. Fuhr
}

\section{TRIENNIAL REPORT 2009-2012}

This report summarizes laboratory measurements of atomic wavelengths, energy levels, hyperfine and isotope structure, energy level lifetimes, and oscillator strengths. Theoretical calculations of lifetimes and oscillator strengths are also included. The bibliography is limited to species of astrophysical interest. Compilations of atomic data and internet databases are also included. Papers are listed in the bibliography in alphabetical order, with a reference number in the text.

\section{Energy levels, wavelengths, line classifications, and line structure}

Major analyses of wavelengths, energy levels and line classifications have been published for V I 257, Fe II [52], [51], Cr I [262], Te II [246], and Ho II [102] in the past three years. Wavelengths and energy levels have also been measured in the symbiotic nova RR Telescopii for the following species: Al VI, Ar III-V, C II, Ca V-VII, Cl IV, K IV-VI, Mg V-VI, N II-III, Na IV-VI, Ne III-V, O IV, P IV, S IV-V, and Si II-IV. Line identifications based on solar flares and active regions were published for Ar XI,XIV, Ca XIV-XV, Fe XII, Fe XIII, Fe XVII, and Ni XV [240].

Additional publications of wavelengths, energy levels and line classifications include:

Al IV-XI [96], Ar II [200], Ba I [298], Ca I [15, 60] IS [229], Ca XI [119], Ce II [162], Cl I [44], Co II HFS [38], Cr I IS [262], Cr III [243], D I [213], Dy I HFS, IS [166], Er I [125], HFS, IS [123], Er II [272], Eu I [274], Fe I HFS, IS [148], Fe II [50, 51, 52, 126], Fe VI-XIV [170], Fe VII-IX [284], Fe VII [286], Fe VIII [287, 159], Fe IX-XVI [173], Fe IX [282, 158], Fe X [134], Fe XI [291, 288, 135], Fe XII-XXII [278], Fe XIII [260], Fe XVII [289, 94], Gd I IS [19, 125], Ge I-II [200], H I IS $[213,21], \mathbf{H g}$ I IS [231], Ho II HFS [102], K I IS, HFS [151, 37], La I HFS [32], La II HFS [86, 87, 88], Li I IS [230], Mg I IS [111, 244], Mg II IS [34], Mg III [46], Mg VII [173], Na I-XI [233], Na I [147], Nb I HFS [149], Nb II HFS [203], O I [121], Os I HFS [33], Pb I-II IS [266, 267], Pr I-II HFS [89], S IX-XIII [279], S V [186], Si I IS, HFS [165], Si VII-IX [173], Sm I [97], Sn I [299], Ta I HFS [95], Ta II HFS [261], Tc I,II [190], Te II [246], Ti I IS, HFS [124], Ti II IS, HFS [204], Ti XIII [119], Tm I HFS [13], V I [257], V XXIII [93], Xe II [285], Zn II [14].

The references for elements heavier than $\mathrm{Ni}(\mathrm{Z}>28)$ are limited to the first three or four spectra only, these data being of most interest for astronomical spectroscopy.

Current analyses of neutral through doubly-ionized spectra are underway for irongroup spectra at the National Institute of Standards and Technology (NIST) and the University of Wisconsin, USA and Imperial College, London, UK. Work on rare-earth elements is being performed at the University of Wisconsin, USA; Laboratoire Aimé 
Cotton, Orsay, France; Observatoire de Paris-Meudon, France; and the Institute of Spectroscopy, Troitsk, Russia. Studies of more highly-ionized elements are being done using electron beam ion traps at NIST, USA, Lawrence Livermore National Laboratory, USA, and Heidelberg, Germany, and with an accelerator in Beijing, China.

\section{Wavelength standards}

Much of the work on wavelength standards during the period of this report has focused on standards required for calibration of astronomical spectrographs and for detecting possible changes in the fine-structure constant during the history of the Universe. Wavelengths emitted by a uranium-neon hollow cathode lamp suitable for astronomical spectrograph calibration have been measured using Fourier transform spectroscopy (FTS) [219]. Updated wavelength standards suitable for detecting changes in the finestructure constant have been measured using FTS for $\mathbf{M g}$ I, Mg II, Ti II, Cr II, Mn II, Fe II and Zn II [14, 200]. The most accurate wavelength standards are now made using laser spectroscopy with a laser frequency comb for calibration. Frequency standards with uncertainties of below $1 \mathrm{MHz}$ have been published using this method for Li I [230], Mg II [34, 112], Ca I [229], and Ca II [271]. Additional laser spectroscopy measurements include the $546 \mathrm{~nm}$ line of ${ }^{198} \mathbf{H g}$ [231], which was a widely used wavelength standard for earlier studies. These laser spectroscopy measurements have been used to validate the scale of FTS measurements [200], putting all of these measurements on the same wavelength scale. Ritz wavelengths based on re-optimized energy levels of ${ }^{198} \mathrm{Hg}$ are found in [145].

\section{Transition probabilities}

The transition-probability data in the references in section 7 were obtained by both theoretical and experimental methods. The references for elements heavier than $\mathrm{Ni}(\mathrm{Z}>28)$ are limited to the first three or four spectra only.

\section{Compilations, Reviews, Conferences}

Major compilations of wavelengths, energy levels or transition probabilities have been published for the following elements: Al [136], Ar [228], B [84, 143, 144], Be [84], Cs [234], H I; D I; T I [146, 269], He [269], K [232], Li [269], Na [233], S [214], Si [137], and Sr I [236]. Additional data can be found in NIST Atomic Transition Probabilities, section of the Handbook of Chemistry and Physics [85].

Papers on atomic spectroscopic data are included in the proceedings of the 10th International Conference on Atomic Spectra and Oscillator Strengths [2], the 7th International Conference on Atomic and Molecular data and their Applications [1], and the 2010 NASA Laboratory Astrophysics workshop [3]. Additional conferences including papers on atomic data include Atomic Processes in Plasmas, the Congress of the European Group on Atomic Systems; and the meeting of the Division of Atomic, Molecular and Optical Physics of the American Physical Society.

\section{Databases}

The following databases of atomic spectra at NIST have received significant updates since the last triennial report: 
NIST Atomic Spectra Database:

http://www.nist.gov:/pml/data/asd.cfm contains critically compiled data on wavelengths, energy levels and oscillators strengths.

Ground Levels and Ionization Energies for the Neutral Atoms:

http://www.nist.gov/pml/data/ion_energy.cfm

NIST Atomic Spectra Bibliographic Databases:

http://www.nist.gov/pml/data/asbib/index.cfm

Consists of three databases of publications on atomic transition probabilities, atomic energy levels and spectra, and atomic spectral line broadening.

Additional on-line databases including significant quantities of atomic data include:

The MCHF/MCDHF Collection on the Web (C.Froese Fischer et al.) at http://nlte.nist.gov/MCHF/index.html contains results of multi-configuration Hartree-Fock (MCHF) or multi-configuration Dirac-Hartree-Fock (MCDHF) calculations for hydrogen and Li-like through Ar-like ions, mainly for $\mathrm{Z} \leqslant 30$. Data for fine-structure transitions are included.

The TOPbase and Opacity Projects include transition probability and oscillator strength data for astrophysically abundant ions $(Z \leqslant 26)$. A database is available at http: //cdsweb.u-strasbg.fr/topbase/topbase.html

CHIANTI, an atomic database for spectroscopic diagnostics of astrophysical plasmas at http://www.chianti.rl.ac.uk/ contains atomic data and programs for computing spectra from astrophysical plasmas, with the emphasis on highly-ionized atoms.

The Vienna Atomic Line Database (VALD) web site (http://ams.astro.univie.ac.at/ vald/) is a collection of atomic line parameters of astronomical interest, with tools for selecting subsets of astrophysical interest.

The bibl database is a comprehensive bibliographic database of experimental and theoretical papers on atomic spectroscopy, with an emphasis on papers published since 1983. It is available at http://das101.isan.troitsk.ru/bibl.htm).

\title{
6. Notes for References
}

The references are identified by a running number. This refers to the general reference list at the end of this report, where the literature is ordered alphabetically according to the first author. Each reference contains one or more code letters indicating the method applied by the authors, defined as follows:

\section{THEORETICAL METHODS:}

Q: quantum mechanical calculations. QF: Calculations of forbidden lines.

\section{EXPERIMENTAL METHODS:}

CL: New classifications

HFS: Hyperfine structure.

TE: Experimental transition probabilities.
EL: Energy levels. WL: Wavelengths.

IS: Isotope structure. L: Lifetimes.

\section{OTHER:}

CP: Data compilations. R: Relative values only. F: Forbidden lines.

\author{
Gillian Nave \\ chair of Working Group
}




\section{References on lifetimes and transition probabilities}

Al II: 56

Al III: 172

Al IV: 168

Al IX: 104

Al V-XII: 212

Al XI: 117

Ar V: 255

Ar VIII: 172

Ar IX: 168

Ar XVI: 117

$\mathrm{Au}$ III: 281

B I: 292

B IV: 6

Ba I: 298

Ba II: 120, 224

Be III: 6

C I: 29

C II: 127,247

C V: 6

Ca I: 15

Ca II: 92, 224, 226

Ca VIII: 132

Ca X: 172

Ca XI: 168

Ca XVII: 154

Ca XVIII: 117

Cd II: 224

Ce I: 59, 108

Ce II: 110, 162

Cl I: 44, 206

Cl VII: 172

Cl VIII: 168

Cl XV: 117
Co XII: : 259

Co XIII: 259

Co XIV: 259

Co XV: 98,259

Co XVII: 172

Co XVIII: 168

Co XXIII: 104

Cr II: 35, 101

Cr VIII: 5

Cr XIV: 172

Cr XV: 168

Cr XXII: 264

Cu II: 48, 76

Er I: 109,164

Er II: 163, 272

Eu I: 294

Eu III: 273

F VII: 12

F VIII: 10

F IX: 11

Fe II: 51, 52, 66, 100, 191, 218

Fe III: 63, 64, 65

Fe IV: 61, 67, 80, 113

Fe VI: 31

Fe VII: 270, 286

Fe VIII: 159,287

Fe IX: 158

Fe X: 43

Fe XI: 259, 288, 291

Fe XII: 250, 252, 258, 259

Fe XIII: 245, 259

Fe XIV: 169, 248, 250, 252, 259

Fe XV: 152, 194

Fe XVI: 172, 198

Fe XVII: 168, 177

Fe XVII-XXV: 114

Fe XIX: 49, 142, 197

Fe XX: 142

Fe XXII: 104, 193, 195

Fe XXIII: 277

Fe XXIV: 178, 263

Fe XXVI: 4, 55
Ga I: 280

Gd I: L 107, 160

Ge IV: 74

He I: 16, 192

Hf I,III: 184

Hg II: 224

In I: 225

K I: $20,54,223$

K II: 254

K IX: 172

K X: 168

K XVII: 117

Kr II: 131

La I: $77,128,129$

La II: 150

Li I-II: 22

Li II: 6

Mg I: 56, 122, 256

Mg II: 69,172

Mg III: 168

Mg IX: 118, 290

Mg X: 117

Mn I: 42

Mn XV: 172

Mn XVI: 168

Mn XXI: 104

Mo II: 180

N I-VII: 90

N I: $27,29,45$

N II: 29, 239, 251

N III: 127

N V: 12

N VI: 10

N VII: 11 
Na I: 53

Na II: 168

Na IX: 12,117

Na X: 10

Na XI: 11

Nb I: 183

Nb II: 202, 203

Nb III: 202

Nd II: 220

Ne I: 25

Ne II: 79,237

Ne VI: 222

Ne VIII: 12

Ne X: 11

Ni XI: 39

Ni XIII: 259

Ni XIV: 156, 259

Ni XV: 259

Ni XVI: 259

Ni XVII: 40

Ni XIX 168

Ni XIX-XXVII: 115

Ni XXIII: 116

Ni XXIV: 104

Ni XXV: 73, 155

O I: $24,28,91,249$

O II: $23,29,141,196$

O III: 81

O IV: 8

O IV: 127

O VII: 7

O VIII: 11

P II: 47, 76

P IV: 56

P V: 172

P VI: 168

P XII: 188

P XIII: 117

Pb III: 18

Pr II-III: 189

Pt II: 217
Rb I: 221

Rh II: 215

$\mathrm{Ru}$ I: 83

$\mathrm{Ru}$ II-III: 210

S I: $26,30,62$

S II: 253

S V: 56

S VI: 172

S VII: 168

S XI-XV: 75

S XIII: 153, 187

S XIV: 117, 179

S XV: 208

Sb I: 106

Sc II: 105, 181

Sc II: 35, 105, 181

Sc III: 227,276

Sc XI: 172

Sc XII: 168

Si I: 174,176

Si II-IV: 72

Si II: 36,72

Si III: 56

Si IV: 172

Si V: 168

Si VI: 103

Si X: 171

Si XI: 268

Si XII: 117

Si XII-XIV: 9

Sm I: 238, 295, 296

Sn I: 17, 275, 297, 299

Sn II: 207

Sn III: 58

Sr II: 224

Ta II: 216

Ta III: 82

Tb I,II: 78
Ti II: 68,209

Ti III-IV: 293

Ti IV: 138, 139

140, 185

Ti X: 241, 242

Ti XII: 172

Ti XIII: 168

V XI: 99

V XIII: 172

V XIV: 168

W II: 201

W III: 211

Xe II: 285

Y II-III: 41

Y III: 227, 276

Yb I: 130

Yb II: 133

Zn I: 71, 175

Zn II: 70, 71

Zr I: 182,199 


\section{References}

[1] 7 th International Conference on Atomic and Molecular Data and their Applications: 2010, AIP Conference Proceedings Volume 1344

[2] 10th International Colloquium on Atomic Spectra and Oscillator Strengths for Astrophysical and Laboratory Plasmas: 2011 Canadian J. Physics, 89

[3] The 2010 NASA Laboratory Astrophysics Workshop: 2011, ed. D.R. Schultz, Oak Ridge National Laboratory http://www-cfadc.phy.ornl.gov/nasa_law/proceedings.html

[4 ] Aggarwal, K. M., Hamada, K., Igarashi, A., Jonauskas, V., Keenan, F. P., \& Nakazaki, S.: 2008, Astron. Astrophys. 484, 879, Q, QF

[5 ] Aggarwal, K. M., Kato, T., Keenan, F. P., \& Murakami, I.: 2009a, Astron. Astrophys. 506, 1501, Q, QF

[6 ] Aggarwal, K. M., Kato, T., Keenan, F. P., \& Murakami, I.: 2011, Phys. Scr. 83, 015302, Q, QF

[7] Aggarwal, K. M. \& Keenan, F. P.: 2008a, Astron. Astrophys. 489, 1377, Q, QF

[8] Aggarwal, K. M. \& Keenan, F. P.: 2008b, Astron. Astrophys. 486 , 1053, Q, QF

[9] Aggarwal, K. M. \& Keenan, F. P.: 2010, Phys. Scr. 82, 065302, Q, QF

[10] Aggarwal, K. M., Keenan, F. P., \& Heeter, R. F.: 2009b, Phys. Scr. 80, 045301, Q, QF

[11] Aggarwal, K. M., Keenan, F. P., \& Heeter, R. F.: 2010a, Phys. Scr. 82, 015006, Q, QF

[12] Aggarwal, K. M., Keenan, F. P., \& Heeter, R. F.: 2010b, Phys. Scr. 81, 015303, Q, QF

[13] Akimov, A. V., Chebakov, K. Y., Tolstikhina, I. Y., Sokolov, A. V., Rodionov, P. B., Kanorsky, S. I., Sorokin, V. N., \& Kolachevsky, N. N.: 2008, Quantum Electron. 38, 961, HFS

[14] Aldenius, M.: 2009, Phys. Scr. T134, 014008, WL, CL

[15] Aldenius, M., Lundberg, H., \& Blackwell-Whitehead, R.: 2009a, Astron. Astrophys. 502, 989, WL, CL, TE, EL

[16] Alexander, S. A. \& Coldwell, R. L.: 2008, Int. J. Quantum Chem. 108, 2813, Q, QF

[17] Alonso-Medina, A.: 2010, Spectrochim. Acta, Part B 65, 158, TE

[18] Alonso-Medina, A., Colón, C., \& Zanón, A.: 2009, Mon. Not. R. Astron. Soc. 395, 567, Q

[19] Ankush, B. K. \& Deo, M. N.: 2010, Phys. Scr. 81, 055301, IS, EL

[20] J. M. P., Serrao, J. M. P. S.: 2008, J. Quant. Spectrosc. Radiat. Transfer 109, 453, Q

[21] Arnoult, O., Nez, F., Julien, L., \& Biraben, F.: 2010, Eur. Phys. J. D 60, 243, WL, EL

[22] Ateş, Ş. \& Çelik, G.: 2009, Acta Phys. Pol. A 116(2), 169, Q

[23] Ateş, Ş., Tekeli, G., Çelik, G., Akin, E., \& Taşer, M.: 2009, Eur. Phys. J. D 54, 21, Q

[24] Bacławski, A.: 2008a, J. Quant. Spectrosc. Radiat. Transfer 109, 1986, TE, R

[25] Bacławski, A.: 2008b, J. Phys. B 41, 225701, TE

[26] Bacławski, A.: 2011, Eur. Phys. J. D 61, 327, TE, R

[27] Bacławski, A. \& Musielok, J.: 2008a, J. Quant. Spectrosc. Radiat. Transfer 109, 2537, TE, $\mathbf{R}$

[28] Bacławski, A. \& Musielok, J.: 2008b, Spectrochim. Acta, Part B 63, 1315, TE

[29] Bacławski, A. \& Musielok, J.: 2009, Acta Phys. Pol. A 116(2), 176, CP

[30] Bacławski, A. \& Musielok, J.: 2011, J. Phys. B 44, 135002, TE, R

[31] Ballance, C. P. \& Griffin, D. C.: 2008, J. Phys. B 41, 195205, Q

[32] Başar, G., Başar, G., \& Kröger, S.: 2009, Opt. Commun. 282, 562, HFS, EL

[33] Başar, G., Başar, G., Kröger, S., \& Guthöhrlein, G. H.: 2010, J. Phys. B 43, 074008, HFS

[34] Batteiger, V., Knünz, S., Herrmann, M., Saathoff, G., Schüssler, H. A., Bernhardt, B., Wilken, T., Holzwarth, R., Hänsch, T. W., \& Udem, T.: 2009, Phys. Rev. A 80, 022503, IS, WL

[35] Bautista, M. A., Ballance, C., Gull, T. R., Hartman, H., Lodders, K., Martínez, M., \& Meléndez, M.: 2009a, Mon. Not. R. Astron. Soc. 393, 1503, QF

[36] Bautista, M. A., Quinet, P., Palmeri, P., Badnell, N. R., Dunn, J., \& Arav, N.: 2009b, Astron. Astrophys. 508, 1527, Q

[37] Behrle, A., Koschorreck, M., \& Köhl, M.: 2011, Phys. Rev. A 83, 052507, IS, HFS

[38] Bergemann, M., Pickering, J. C., \& Gehren, T.: 2010, Mon. Not. R. Astron. Soc. 401, 1334, HFS 
[39] Bhatia, A. K. \& Landi, E.: 2011a, At. Data Nucl. Data Tables 97, 50, Q, QF

[40] Bhatia, A. K. \& Landi, E.: 2011b, At. Data Nucl. Data Tables 97, 189, Q, QF

[41] Biémont, E., Blagoev, K., Engström, L., Hartman, H., Lundberg, H., Malcheva, G., Nilsson, H., Whitehead, R. B., Palmeri, P., \& Quinet, P.: 2011, Mon. Not. R. Astron. Soc. 414, 3350, L, Q

[42] Blackwell-Whitehead, R., Pavlenko, Y. V., Nave, G., Pickering, J. C., Jones, H. R. A., Lyubchik, Y., \& Nilsson, H.: 2011, Astron. Astrophys. 525, p. A44, TE

[43] Brenner, G., López-Urrutia, J. R. C., Bernitt, S., Fischer, D., Ginzel, R., Kubiček, K., Mäckel, V., Mokler, P. H., Simon, M. C., \& Ullrich, J.: 2009, Astrophys. J. 703, 68, LF

[44] Bridges, J. M. \& Wiese, W. L.: 2008a, Phys. Rev. A 78, 062508, TE

[45] Bridges, J. M. \& Wiese, W. L.: 2010, Phys. Rev. A 82, 024502, TE

[46] Brown, C. M., Kramida, A. E., Feldman, U., \& Reader, J.: 2009a, Phys. Scr. 80, 065302, EL, CL, WL

[47] Brown, J. B., Brown, M. S., Cheng, S., Curtis, L. J., Ellis, D. G., Federman, S. R., \& Irving, R. E.: 2011, Can. J. Phys. 89, 413, Q, R

[48] Brown, M. S., Federman, S. R., Irving, R. E., Cheng, S., \& Curtis, L. J.: 2009b, Astrophys. J. $702,880, \mathbf{L}$

[49] Butler, K. \& Badnell, N. R.: 2008, Astron. Astrophys. 489, 1369, Q

[50] Castelli, F., Johansson, S., \& Hubrig, S.: 2008, J. Phys.: Conf. Ser. 130, 012003, CL, WL

[51] Castelli, F. \& Kurucz, R. L.: 2010a, Astron. Astrophys. 520, p. A57, EL, CL, Q

[52] Castelli, F., Kurucz, R. L., \& Hubrig, S.: 2009a, Astron. Astrophys. 508, 401, CL, WL, EL, Q

[53] Çelik, G. \& Ateş, Ş.: 2008a, Acta Phys. Pol. A 113(6), 1619, Q

[54] Çelik, G. \& Ateş, Ş.: 2008b, Can. J. Phys. 86, 487, Q

[55] Chen, C.-Y., Wang, K., Huang, M., Wang, Y.-S., \& Zou, Y.-M.: 2010, J. Quant. Spectrosc. Radiat. Transfer 111, 843, Q, QF

[56] Cheng, C., Gao, X., Qing, B., Zhang, X.-L., \& Li, J.-M.: 2011, Chin. Phys. B 20, 033103, Q

[57] Chwalla, M., Benhelm, J., Kim, K., Kirchmair, G., Monz, T., Riebe, M., Schindler, P., Villar, A. S., Hänsel, W., Roos, C. F., Blatt, R., Abgrall, M., Santarelli, G., Rovera, G. D., \& Laurent, P.: 2009, Phys. Rev. Lett. 102, 023002, WL

[58] Colón, C. \& Alonso-Medina, A.: 2010, J. Phys. B 43, 165001, Q

[59] Curry, J. J.: 2009, J. Phys. D 42, 135205, TE

[60] Dammalapati, U., Norris, I., Burrows, C., \& Riis, E.: 2011, Phys. Rev. A 83, 062513, CL, WL

[61] Deb, N. C. \& Hibbert, A.: 2008a, J. Phys. B 41, 081007, Q

[62] Deb, N. C. \& Hibbert, A.: 2008b, At. Data Nucl. Data Tables 94, 561, Q

[63] Deb, N. C. \& Hibbert, A.: 2008c, J. Phys.: Conf. Ser. 130, 012006, Q, QF

[64] Deb, N. C. \& Hibbert, A.: 2009a, J. Phys. B 42, 065003, QF

[65] Deb, N. C. \& Hibbert, A.: 2009b, At. Data Nucl. Data Tables 95, 184, Q

[66] Deb, N. C. \& Hibbert, A.: 2010a, Astrophys. J. 711, L104, QF

[67] Deb, N. C. \& Hibbert, A.: 2010b, At. Data Nucl. Data Tables 96, 358, Q

[68] Deb, N. C., Hibbert, A., Felfli, Z., \& Msezane, A. Z.: 2009, J. Phys. B 42, 015701, QF

[69] Destree, J. D., Williamson, K. E., \& Snow, T. P.: 2010a, Astrophys. J. 712, L48, CL, WL, TE

[70] Dixit, G., Nataraj, H. S., Sahoo, B. K., Chaudhuri, R. K., \& Majumder, S.: 2008, J. Phys. B 41, 025001, Q, QF

[71] Dixit, G., Sahoo, B. K., Chaudhuri, R. K., \& Majumder, S.: 2009, J. Phys. B 42, 165702, Q, QF

[72] Djeniže, S., Srećković, A., \& Bukvić, S.: 2010, Spectrochim. Acta, Part B 65, 61, TE, R

[73] Duan, B., Bari, M. A., Zhong, J. Y., Yan, J., Li, Y. M., \& Zhang, J.: 2008, Astron. Astrophys. $488,1155, \mathbf{Q}, \mathbf{Q F}$

[74] Dutta, N. N. \& Majumder, S.: 2011, Astrophys. J. 737, 25, Q, QF

[75] Fan, Q., Liao, Z. J., Yang, J. H., \& Zhang, J. P.: 2009, Phys. Scr. 79, 015301, Q 
[76] Federman, S. R., Curtis, L. J., Brown, M., Cheng, S., Irving, R. E., Torok, S., \& Schectman, R. M.: 2008, J. Phys.: Conf. Ser. 130, 012007, TE, R, L

[77] Feng, Y.-Y., Zhang, W., Kuang, B., Ning, L.-L., Jiang, Z.-K., \& Dai, Z.-W.: 2011, J. Opt. Soc. Am. B 28, 543, L

[78] Feng, Y.-Y., Zhang, W., Ning, L.-L., Kuang, B., Sun, G.-J., \& Dai, Z.-W.: 2010, J. Phys. B 43, 225001, L

[79] Fischer, C. F. \& Ralchenko, Y.: 2008, Int. J. Mass Spectrom. 271, 85, Q

[80] Fischer, C. F., Rubin, R. H., \& Rodríguez, M.: 2008, Mon. Not. R. Astron. Soc. 391 , 1828, QF

[81] Fischer, C. F., Tachiev, G., Rubin, R. H., \& Rodríguez, M.: 2009, Astrophys. J. 703, 500, $\mathrm{Q}, \mathrm{QF}$

[82] Fivet, V., Biémont, E., Engström, L., Lundberg, H., Nilsson, H., Palmeri, P., \& Quinet, P.: 2008, J. Phys. B 41, 015702, L, Q

[83] Fivet, V., Quinet, P., Palmeri, P., Biémont, E., Asplund, M., Grevesse, N., Sauval, A. J., Engström, L., Lundberg, H., Hartman, H., \& Nilsson, H.: 2009, Mon. Not. R. Astron. Soc. $396,2124, \mathbf{L}, \mathbf{Q}$

[84] Fuhr, J. R. \& Wiese, W. L.: 2010, J. Phys. Chem. Ref. Data 39, 013101, CP

[85] Fuhr, J. R. \& Wiese, W. L.: 2011, CRC Handbook of Chemistry and Physics, 92nd Edition, (Ed. D. R. Lide) CRC Press, Boca Raton, FL,

[86] Furmann, B., Elantkowska, M., Stefańska, D., Ruczkowski, J., \& Dembczyński, J.: 2008a, J. Phys. B 41, 235002, HFS

[87] Furmann, B., Ruczkowski, J., Stefańska, D., Elantkowska, M., \& Dembczyński, J.: 2008b, J. Phys. B 41, 215004, HFS

[88] Furmann, B., Stefańska, D., \& Dembczyński, J.: 2010, J. Phys. B 43, 015001, EL,CL, WL, HFS

[89] Gamper, B., Uddin, Z., Jahangir, M., Allard, O., Knöckel, H., Tiemann, E., \& Windholz, L.: 2011, J. Phys. B 44, 045003, CL, WL, EL,HFS

[90] García, J., Kallman, T. R., Witthoeft, M., Behar, E., Mendoza, C., Palmeri, P., Quinet, P., Bautista, M. A., \& Klapisch, M.: 2009, Astrophys. J., Suppl. Ser. 185, 477, Q

[91] Gattinger, R. L., Lloyd, N. D., Bourassa, A. E., Degenstein, D. A., McDade, I. C., \& Llewellyn, E. J.: 2009, Can. J. Phys. 87, 1133, TE-R-F

[92] Gerritsma, R., Kirchmair, G., Zähringer, F., Benhelm, J., Blatt, R., \& Roos, C. F.: 2008, Eur. Phys. J. D 50, 13, TE

[93] Gillaspy, J. D., Chantler, C. T., Paterson, D., Hudson, L. T., Serpa, F. G., \& Takács, E.: 2010, J. Phys. B 43, 074021, CL, EL,WL

[94] Gillaspy, J. D., Lin, T., Tedesco, L., Tan, J. N., Pomeroy, J. M., Laming, J. M., Brickhouse, N., Chen, G.-X., \& Silver, E.: 2011, Astrophys. J. 728, 132, WL

[95] Głowacki, P., Uddin, Z., Guthöhrlein, G. H., Windholz, L., \& Dembczyński, J.: 2009, Phys. Scr. 80, 025301, CL, WL, EL, HFS

[96] Gu, M. F., Beiersdorfer, P., \& Lepson, J. K.: 2011, Astrophys. J. 732, 91, CL, WL

[97] Guan, F., Dai, C.-J., \& Zhao, H.-Y.: 2008, Chin. Phys. B 17, 3655, EL, CL, WL

[98] Gupta, G. P. \& Msezane, A. Z.: 2008, Eur. Phys. J. D 49, 157, Q

[99] Gupta, G. P. \& Msezane, A. Z.: 2010, Phys. Scr. 81, 045302, Q

[100] Gurell, J., Hartman, H., Blackwell-Whitehead, R., Nilsson, H., Bäckström, E., Norlin, L. O., Royen, P., \& Mannervik, S.: 2009a, Astron. Astrophys. 508, 525, L, F

[101] Gurell, J., Nilsson, H., Engström, L., Lundberg, H., Blackwell-Whitehead, R., Nielsen, K. E., \& Mannervik, S.: 2010, Astron. Astrophys. 511, p. A68, L, TE

[102] Gurell, J., Wahlgren, G. M., Nave, G., \& Wyart, J.-F.: 2009b, Phys. Scr. 79, 035306, CL, WL, HFS, EL

[103] Hamdi, R., Nessib, N. B., Milovanović, N., Popović, L. Č., Dimitrijević, M. S., \& Sahal-Bréchot, S.: 2008, Mon. Not. R. Astron. Soc. 387, 871, Q

[104] Hao, L.-H. \& Jiang, G.: 2011, Phys. Rev. A 83, 012511, Q

[105] Hartman, H., Gurell, J., Lundin, P., Schef, P., Hibbert, A., Lundberg, H., Mannervik, S., Norlin, L.-O., \& Royen, P.: 2008, Astron. Astrophys. 480, 575, LF, QF

[106] Hartman, H., Nilsson, H., Engström, L., Lundberg, H., Palmeri, P., Quinet, P., \& Biémont, E.: 2010, Phys. Rev. A 82, 052512, L, Q 
[107] Hartog, E. A. D., Bilty, K. A., \& Lawler, J. E.: 2011, J. Phys. B 44, 055001, L

[108] Hartog, E. A. D., Buettner, K. P., \& Lawler, J. E.: 2009, J. Phys. B 42, 085006, L

[109] Hartog, E. A. D., Chisholm, J. P., \& Lawler, J. E.: 2010, J. Phys. B 43, 155004, L

[110] Hartog, E. A. D. \& Lawler, J. E.: 2008, J. Phys. B 41, 045701, L

[111] He, M., Therkildsen, K. T., Jensen, B. B., Brusch, A., Thomsen, J. W., \& Porsev, S. G.: 2009, Phys. Rev. A 80, 024501, IS, HFS

[112] Herrmann, Batteiger, M. V., Knünz S., Saathoff G., Udem Th., and Hänsch T. W., 2009, Phys. Rev. Lett. 102, 013006 WL

[113] Hibbert, A. \& Deb, N. C.: 2008, J. Phys.: Conf. Ser. 130, 012012, Q

[114] Hou, H.-J., Jiang, G., Hu, F., \& Hao, L.-H.: 2009, At. Data Nucl. Data Tables 95, 125, Q

[115] Hu, F., Jiang, G., Hong, W., \& Hao, L. H.: 2008, Eur. Phys. J. D 49, 293, Q

[116] Hu, F., Jiang, G., Yang, J. M., Zhang, J. Y., \& Zhao, X. F.: 2011, Acta Phys. Pol. A $120(3), 429, \mathbf{Q}$

[117] Hu, M.-H. \& Wang, Z.-W.: 2009, Chin. Phys. B 18, 2244, Q

[118] Hudson, C. E.: 2009, Astron. Astrophys. 493, 697, Q

[119] Ishikawa, Y., Encarnación, J. M. L., \& Träbert, E.: 2009, Phys. Scr. 79, 025301, Q

[120] Iskrenova-Tchoukova, E. \& Safronova, M. S.: 2008, Phys. Rev. A 78, 012508, Q, QF

[121] Ivanov, T. I., Salumbides, E. J., Vieitez, M. O., Cacciani, P. C., de Lange, C. A., \& Ubachs, W.: 2008, Mon. Not. R. Astron. Soc. 389, L4, WL, CL

[122] Jensen, B. B., Ming, H., Westergaard, P. G., Gunnarsson, K., Madsen, M. H., Brusch, A., Hald, J., \& Thomsen, J. W.: 2011, Phys. Rev. Lett. 107, 113001, LF

[123] Jin, W.-G., Nakai, H., Kawamura, M., \& Minowa, T.: 2009a, J. Phys. Soc. Jpn. 78, 015001, HFS, IS

[124] Jin, W.-G., Nemoto, Y., \& Minowa, T.: 2009b, J. Phys. Soc. Jpn. 78, 094301, IS, HFS

[125] Jin, W.-G., Nemoto, Y., Nakai, H., Kawamura, M., \& Minowa, T.: 2008, J. Phys. Soc. Jpn. 77, 124301, IS

[126] Johansson, S.: 2009, Phys. Scr. T134, 014013, EL, CL

[127] Jönsson, P., Li, J.-G., Gaigalas, G., \& Dong, C.-Z.: 2010, At. Data Nucl. Data Tables 96, $271, \mathbf{Q}$

[128] Karaçoban, B. \& Özdemir, L.: 2008a, J. Quant. Spectrosc. Radiat. Transfer 109, 1968, Q

[129] Karaçoban, B. \& Özdemir, L.: 2008b, Acta Phys. Pol. A 113(6), 1609, Q

[130] Karaçoban, B. \& Özdemir, L.: 2011, J. Kor. Phys. Soc. 58, 417, Q

[131] Karmakar, S. \& Das, M. B.: 2010, Eur. Phys. J. D 59, 361, L

[132] Karpuškienè, R. \& Bogdanovich, P.: 2009, At. Data Nucl. Data Tables 95, 533, Q

[133] Kedzierski, D., Kusz, J., \& Muzolf, J.: 2010, Spectrochim. Acta, Part B 65, 248, TE

[134] Keenan, F. P., Jess, D. B., Aggarwal, K. M., Thomas, R. J., Brosius, J. W., \& Davila, J. M.: 2008a, Mon. Not. R. Astron. Soc. 389, 939, CL, Q, QF

[135] Keenan, F. P., Milligan, R. O., Jess, D. B., Aggarwal, K. M., Mathioudakis, M., Thomas, R. J., Brosius, J. W., \& Davila, J. M.: 2010, Mon. Not. R. Astron. Soc. 404, 1617, CL, WL

[136] Kelleher, D. E. \& Podobedova, L. I.: 2008a, J. Phys. Chem. Ref. Data 37, 709, CP

[137] Kelleher, D. E. \& Podobedova, L. I.: 2008b, J. Phys. Chem. Ref. Data 37, 1285, CP

[138] Kingston, A. E. \& Hibbert, A.: 2008, J. Phys. B 41, 155001, Q

[139] Kingston, A. E. \& Hibbert, A.: 2009, J. Phys. B 42, 185004, Q

[140] Kingston, A. E. \& Hibbert, A.: 2010, J. Phys. B 43, 165003, Q

[141] Kisielius, R., Storey, P. J., Ferland, G. J., \& Keenan, F. P.: 2009, Mon. Not. R. Astron. Soc. 397, 903, Q

[142] Kotochigova, S., Linnik, M., Kirby, K. P., \& Brickhouse, N. S.: 2010, Astrophys. J., Suppl. Ser. 186, 85, Q

[143] Kramida, A., Ryabtsev, A. N., \& Ekberg, J. O., Kink, I., Mannervik, S., Martinson, I.: 2008, Phys. Scr. 78, 025301 CP

[144] Kramida, A., Ryabtsev, A. N., Ekberg, J. O., Kink, I., Mannervik, S., Martinson, I.: 2008 Phys. Scr. 78, 025302 CP

[145] Kramida, A.: 2011a, J. Res. Natl. Inst. Stand. Technol. 116(2), 599, EL, CL, WL 
[146] Kramida, A. E.: 2010a, At. Data Nucl. Data Tables 96, 586, E, WL, CL, HFS, QF

[147] Kramida, A. E.: 2010b, J. Phys. B 43, 205001, EL,WL, CL

[148] Krins, S., Oppel, S., Huet, N., von Zanthier, J., \& Bastin, T.: 2009, Phys. Rev. A 80, 062508, IS, HFS

[149] Kröger, S., Er, A., Öztürk, I. K., Başar, G., Jarmola, A., Ferber, R., Tamanis, M., \& Začs, L.: 2010, Astron. Astrophys. 516, p. A70, HFS

[150] Kułaga-Egger, D. \& Migdałek, J.: 2009, J. Phys. B 42, 185002, Q

[151] Kumar, P. V. K. \& Suryanarayana, M. V.: 2011, J. Phys. B 44, 055003, IS, HFS, WL

[152] Landi, E.: 2011, At. Data Nucl. Data Tables 97, 587, Q

[153] Landi, E. \& Bhatia, A. K.: 2008, At. Data Nucl. Data Tables 94, 1, Q, QF

[154] Landi, E. \& Bhatia, A. K.: 2009a, At. Data Nucl. Data Tables 95, 155, Q, QF

[155] Landi, E. \& Bhatia, A. K.: 2009b, At. Data Nucl. Data Tables 95, 547, Q, QF

[156] Landi, E. \& Bhatia, A. K.: 2010, At. Data Nucl. Data Tables 96, 52, Q, QF

[157] Landi, E. \& Young, P. R.: 2009a, Astrophys. J. 706, 1, DB

[158] Landi, E. \& Young, P. R.: 2009b, Astrophys. J. 707, 1191, CL, WL, EL,Q

[159] Landi, E. \& Young, P. R.: 2010a, Astrophys. J. 713, 205, CL, WL, EL,Q

[160] Lawler, J. E., Bilty, K. A., \& Hartog, E. A. D.: 2011, J. Phys. B 44, 095001, TE

[161] Lawler, J. E., Chisholm, J., Nitz, D. E., Wood, M. P., Sobeck, J., \& Hartog, E. A. D.: 2010a, J. Phys. B 43, 085701, TE

[162] Lawler, J. E., Sneden, C., Cowan, J. J., Ivans, I. I., \& Hartog, E. A. D.: 2009a, Astrophys. J., Suppl. Ser. 182, 51, L, TE

[163] Lawler, J. E., Sneden, C., Cowan, J. J., Wyart, J.-F., Ivans, I. I., Sobeck, J. S., Stockett, M. H., \& Hartog, E. A. D.: 2008b, Astrophys. J., Suppl. Ser. 178, 71, TE

[164] Lawler, J. E., Wyart, J.-F., \& Hartog, E. A. D.: 2010b, J. Phys. B 43, 235001, TE

[165] Lee, S. A. and Jr., W. M. F.: 2010, Phys. Rev. A 82, 042515, IS, HFS

[166] Leefer, N., Cingöz, A., \& Budker, D.: 2009, Opt. Lett. 34, 2548, IS, HFS, EL

[167] Li, H.-L., Li, P., Cheng, Z., \& Ma, H.-R.: 2008, Commun. Theor. Phys. 49, 217, Q

[168] Liang, G. Y. \& Badnell, N. R.: 2010, Astron. Astrophys. 518, p. A64, Q

[169] Liang, G. Y., Badnell, N. R., López-Urrutia, J. R. C., Baumann, T. M., Zanna, G. D., Storey, P. J., Tawara, H., \& Ullrich, J.: 2010, Astrophys. J., Suppl. Ser. 190, 322, Q

[170] Liang, G. Y., Baumann, T. M., López-Urrutia, J. R. C., Epp, S. W., Tawara, H., Gonchar, A., Mokler, P. H., Zhao, G., \& Ullrich, J.: 2009a, Astrophys. J. 696, 2275, CL

[171] Liang, G. Y., Whiteford, A. D., \& Badnell, N. R.: 2009b, Astron. Astrophys. 499, 943, Q

[172] Liang, G. Y., Whiteford, A. D., \& Badnell, N. R.: 2009c, Astron. Astrophys. 500, 1263, Q

[173] Liang, G. Y. \& Zhao, G.: 2010, Mon. Not. R. Astron. Soc. 405, 1987, WL, CL

[174] Liang, L., Jiang, W. X., Zhou, C., \& Zhang, L.: 2008a, Opt. Commun. 281, 2107, Q

[175] Liang, L. \& Zhou, C.: 2008, J. Quant. Spectrosc. Radiat. Transfer 109 , 1995, Q

[176] Liang, L., Zhou, C., \& Zhang, L.: 2008c, Chin. Opt. Lett. 6, 804, Q

[177] López-Urrutia, J. R. C. \& Beiersdorfer, P.: 2010, Astrophys. J. 721, 576, EF, LF

[178] Louzon, E., Feigel, A., Frank, Y., Raicher, E., Klapisch, M., Mandelbaum, P., Levy, I., Hurvitz, G., Ehrlich, Y., Frankel, M., Maman, S., \& Henis, Z.: 2011, High En. Dens. Phys. $7,124, \mathbf{Q}$

[179] Luna, F. R. T., Mania, A. J., \& Hernandes, J. A.: 2009, J. Appl. Spectrosc. 76, 447, Q

[180] Lundberg, H., Engström, L., Hartman, H., Nilsson, H., Palmeri, P., Quinet, P., \& Biémont, E.: 2010, J. Phys. B 43, 085004, L, TE, Q

[181] Lundin, P., Gurell, J., Mannervik, S., Royen, P., Norlin, L.-O., Hartman, H., \& Hibbert, A.: 2008, Phys. Scr. 78, 015301, LF, QF

[182] Malcheva, G., Mayo, R., Ortiz, M., Ruiz, P., Engström, L., Lundberg, H., Nilsson, H., Quinet, P., Biémont, E., \& Blagoev, K.: 2009a, Mon. Not. R. Astron. Soc. 395, 1523, L, $\mathbf{Q}$

[183] Malcheva, G., Nilsson, H., Engström, L., Lundberg, H., Biémont, E., Palmeri, P., Quinet, P., \& Blagoev, K.: 2011, Mon. Not. R. Astron. Soc. 412, 1823, L, Q

[184] Malcheva, G., Yoca, S. E., Mayo, R., Ortiz, M., Engström, L., Lundberg, H., Nilsson, H., Biémont, E., \& Blagoev, K.: 2009b, Mon. Not. R. Astron. Soc. 396, 2289, L, Q 
[185] Mandal, S., Dixit, G., Sahoo, B. K., Chaudhuri, R. K., \& Majumder, S.: 2008, J. Phys. B 41, 055701, Q, QF

[186] Mania, A. J., Luna, F. R. T., Borges, F. O., \& Cavalcanti, G. H.: 2009a, J. Quant. Spectrosc. Radiat. Transfer 110, 2162, EL, CL, WL, Q

[187] Mania, A. J., Luna, F. R. T., \& Hernandes, J. A.: 2009c, J. Quant. Spectrosc. Radiat. Transfer 110, 82, Q

[188] Mania, A. J., Luna, F. R. T., \& Mania, E.: 2011, J. Appl. Spectrosc. 77, 758, Q

[189] Mashonkina, L., Ryabchikova, T., Ryabtsev, A., \& Kildiyarova, R.: 2009, Astron. Astrophys. 495, 297, Q

[190] Mattolat, C., Gottwald, T., Raeder, S., Rothe, S., Schwellnus, F., Wendt, K., ThörlePospiech, P., \& Trautmann, N.: 2010, Phys. Rev. A 81, 052513, EL

[191] Meléndez, J. \& Barbuy, B.: 2009, Astron. Astrophys. 497, 611, TE, Q

[192] Morton, D. C. \& Drake, G. W. F.: 2011, Phys. Rev. A 83, 042503, Q, QF

[193] Nahar, S. N.: 2008, J. Quant. Spectrosc. Radiat. Transfer 109 , 2731, Q

[194] Nahar, S. N.: 2009, At. Data Nucl. Data Tables 95, 577, Q, QF

[195] Nahar, S. N.: 2010a, At. Data Nucl. Data Tables 96, 26, Q, QF

[196] Nahar, S. N.: 2010b, At. Data Nucl. Data Tables 96, 863, Q

[197] Nahar, S. N.: 2011, At. Data Nucl. Data Tables 97, 403, Q, QF

[198] Nahar, S. N., Eissner, W., Sur, C., \& Pradhan, A. K.: 2009, Phys. Scr. 79, 035401, Q, QF

[199] Nakhate, S. G., Mukund, S., \& Bhattacharyya, S.: 2010, J. Quant. Spectrosc. Radiat. Transfer 111, 394, L

[200] Nave, G. \& Sansonetti, C. J.: 2011, J. Opt. Soc. Am. B 28, 737, WL, EL

[201] Nilsson, H., Engström, L., Lundberg, H., Palmeri, P., Fivet, V., Quinet, P., \& Biémont, E.: 2008, Eur. Phys. J. D 49, 13, L, Q

[202] Nilsson, H., Hartman, H., Engström, L., Lundberg, H., Sneden, C., Fivet, V., Palmeri, P., Quinet, P., \& Biémont, E.: 2010, Astron. Astrophys. 511, p. A16, L, TE, Q, T

[203] Nilsson, H. \& Ivarsson, S.: 2008a, Astron. Astrophys. 492, 609, HFS, TE

[204] Nouri, Z., Rosner, S. D., Li, R., Scholl, T. J., \& Holt, R. A.: 2010, Phys. Scr. 81, 065301, IS, HFS

[205] Oliver, P. \& Hibbert, A.: 2008a, J. Phys. B 41, 165003, Q

[206] Oliver, P. \& Hibbert, A.: 2008b, J. Phys.: Conf. Ser. 130, 012016, Q

[207] Oliver, P. \& Hibbert, A.: 2010, J. Phys. B 43, 074013, Q

[208] Özdemir, L., Ürer, G., \& Karaçoban, B.: 2008, J. Quant. Spectrosc. Radiat. Transfer 109 , 1886, Q

[209] Palmeri, P., Quinet, P., Biémont, E., Gurell, J., Lundin, P., Norlin, L.-O., Royen, P., Blagoev, K., \& Mannervik, S.: 2008, J. Phys. B 41, 125703, LF, QF

[210] Palmeri, P., Quinet, P., Fivet, V., Biémont, E., Cowley, C. R., Engström, L., Lundberg, H., Hartman, H., \& Nilsson, H.: 2009, J. Phys. B 42, 165005, L, Q

[211] Palmeri, P., Quinet, P., Fivet, V., Biémont, E., Nilsson, H., Engström, L., \& Lundberg, H.: 2008, Phys. Scr. 78, 015304, L, Q

[212] Palmeri, P., Quinet, P., Mendoza, C., Bautista, M. A., García, J., Witthoeft, M. C., \& Kallman, T. R.: 2011, Astron. Astrophys. 525, p. A59, Q

[213] Parthey, C. G., Matveev, A., Alnis, J., Pohl, R., Udem, T., Jentschura, U. D., Kolachevsky, N., \& Hänsch, T. W.: 2010, Phys. Rev. Lett. 104, p. 233001, IS, EL, WL

[214] Podobedova, L. I., Kelleher, D. E., \& Wiese, W. L.: 2009, J. Phys. Chem. Ref. Data 38, $171, \mathbf{C P}$

[215] Quinet, P., Biémont, E., Palmeri, P., Engström, L., Hartman, H., Lundberg, H., \& Nilsson, H.: 2011, J. Electron Spectrosc. Relat. Phenom. 184, 174, Q

[216] Quinet, P., Fivet, V., Palmeri, P., Biémont, E., Engström, L., Lundberg, H., \& Nilsson, H.: 2009, Astron. Astrophys. 493, 711, Q, L

[217] Quinet, P., Palmeri, P., Fivet, V., Biémont, E., Nilsson, H., Engström, L., \& Lundberg, H.: 2008, Phys. Rev. A 77, 022501, L, Q

[218] Ramsbottom, C. A.: 2009, At. Data Nucl. Data Tables 95, 910, Q

[219] Redman, S. L., Lawler, J. E., Nave, G., Ramsey, L. W., \& Mahadevan, S.: 2011, Astrophys. J., Suppl. Ser. 195, 24, CL, WL 
[220] Rehse, S. J. \& Ryder, C. A.: 2009, Spectrochim. Acta, Part B 64, 974, TE

[221] Safronova, M. S. \& Safronova, U. I.: 2011a, Phys. Rev. A 83, 052508, Q, QF

[222] Safronova, U. I. \& Mancini, R.: 2009, At. Data Nucl. Data Tables 95, 54, Q

[223] Safronova, U. I. \& Safronova, M. S.: 2008, Phys. Rev. A 78, 052504, Q

[224] Safronova, U. I. \& Safronova, M. S.: 2011b, Can. J. Phys. 89, 465, Q, QF

[225] Sahoo, B. K. \& Das, B. P.: 2011, Phys. Rev. A 84, 012501, Q, QF

[226] Sahoo, B. K., Das, B. P., \& Mukherjee, D.: 2009, Phys. Rev. A 79, 052511, Q

[227] Sahoo, B. K., Nataraj, H. S., Das, B. P., Chaudhuri, R. K., \& Mukherjee, D.: 2008, J. Phys. B 41, 055702, QF

[228] Saloman, E. B.: 2010, J. Phys. Chem. Ref. Data 39, 033101, CP

[229] Salumbides, E. J. , Maslinskas, V., Dildar, U. M., Wolf, A. L., van Duijn, E.-J., Eikema, K. S. E., Ubachs, W.: 2011 Phys. Rev. A 83, 012502 WL

[230] Sansonetti, C. J., Simien, C. E., Gillaspy, J. D., Tan, J. N., Brewer, S. M., Brown, R. C., Wu, S.-J., \& Porto, J. V.: 2011, Phys. Rev. Lett. 107, 023001, WL, HFS, IS, EL

[231 Sansonetti, C. J. \& Veza, D.: 2010, J. Phys. B 43, 205003, WL, IS, HFS

[232] Sansonetti, J. E.: 2008a, J. Phys. Chem. Ref. Data 37, 7, CP

[233] Sansonetti, J. E.: 2008b, J. Phys. Chem. Ref. Data 37, 1659, CP

[234] Sansonetti, J. E.: 2009a, J. Phys. Chem. Ref. Data 38, 761, CP

[235] Sansonetti, J. E.: 2009b, J. Phys. Chem. Ref. Data 38, 761, CP

[236 Sansonetti, J. E. \& Nave, G.: 2010a, J. Phys. Chem. Ref. Data 39, 033103, CP

[237] Santos, J. P., Costa, A. M., Madruga, C., Parente, F., \& Indelicato, P.: 2011, Eur. Phys. J. D $63,89, \mathbf{Q}$

[238] Shah, M. L., Pulhani, A. K., Gupta, G. P., \& Suri, B. M.: 2010, J. Opt. Soc. Am. B 27, $423, \mathbf{L}, \mathbf{T E}$

[239] Shen, X.-Z., Yuan, P., \& Liu, J.: 2010, Chin. Phys. B 19, 053101, Q, QF

[240] Shestov, S. V., Bozhenkov, S. A., Zhitnik, I. A., Kuzin, S. V., Urnov, A. M., Beigman, I. L., Goryaev, F. F., \& Tolstikhina, I. Y.: 2008, Astron. Lett. 34, 33, CL, WL

[241] Singh, J., Jha, A. K. S., \& Mohan, M.: 2010a, J. Phys. B 43, 115005, Q

[242] Singh, J., Jha, A. K. S., Verma, N., \& Mohan, M.: 2010b, At. Data Nucl. Data Tables 96, $759, \mathbf{Q}$

[243] Smillie, D. G., Pickering, J. C., \& Smith, P. L.: 2008, Mon. Not. R. Astron. Soc. 390, 733, WL, CL

[244] Steenstrup, M. P., Brusch, A., Jensen, B. B., Hald, J., \& Thomsen, J. W.: 2010, Phys. Rev. A 82, 054501, IS

[245] Storey, P. J. \& Zeippen, C. J.: 2010, Astron. Astrophys. 511, p. A78, Q

[246] Tauheed, A., Joshi, Y. N., \& Steinitz, M.: 2009, Can. J. Phys. 87, 1255, WL, CL, EL

[247] Tayal, S. S.: 2008a, Astron. Astrophys. 486, 629, Q

[248] Tayal, S. S.: 2008b, Astrophys. J., Suppl. Ser. 178, 359, Q

[249] Tayal, S. S.: 2009a, Phys. Scr. 79, 015303, Q

[250] Tayal, S. S.: 2009b, Phys. Rev. A 80, 032512, QF

[251] Tayal, S. S.: 2011a, Phys. Rev. A 83, 012515, Q, QF

[252] Tayal, S. S.: 2011b, At. Data Nucl. Data Tables 97, 481, Q

[253] Tayal, S. S. \& Zatsarinny, O.: 2010a, Astrophys. J., Suppl. Ser. 188, 32, Q, QF

[254] Tayal, S. S. \& Zatsarinny, O.: 2010b, Astron. Astrophys. 510, p. A79, Q

[255] Tayal, V., Gupta, G. P., \& Tripathi, A. N.: 2009, Indian J. Phys. 83, 1271, Q

[256] Therkildsen, K. T., Jensen, B. B., Ryder, C. P., Malossi, N., \& Thomsen, J. W.: 2009, Phys. Rev. A 79, 034501, TE

[257] Thorne, A. P., Pickering, J. C., \& Semeniuk, J.: 2011, Astrophys. J., Suppl. Ser. 192, 11, CL, WL, EL

[258] Träbert, E., Hoffman, J., Reinhardt, S., Wolf, A., \& Zanna, G. D.: 2008, J. Phys.: Conf. Ser. 130, 012018, LF

[259] Träbert, E., Hoffmann, J., Krantz, C., Wolf, A., Ishikawa, Y., \& Santana, J. A.: 2009, J. Phys. B 42, 025002, LF

[260] Träbert, E., Ishikawa, Y., Santana, J. A., \& Zanna, G. D.: 2011, Can. J. Phys. 89, 403, EL, CL, TE 
[261 Uddin, Z. and Windholz, L.: 2009, Chin. J. Phys. 47(4), 454, HFS, CL, WL

[262] Wallace, L. \& Hinkle, K.: 2009, Astrophys. J. 700, 720, CL, WL, EL

[263] Wang, Z.-W., Li, X.-R., Hu, M.-H., Liu, Y., \& Wang, Y.-N.: 2008a, Chin. Phys. Lett. 25, $2004, \mathbf{Q}$

[264] Wang, Z.-W., Liu, Y., Hu, M.-H., Li, X.-R., \& Wang, Y.-N.: 2008b, Chin. Phys. B 17, $2909, \mathbf{Q}$

[265] Wang, Z.-W., Wang, Y.-N., Hu, M.-H., Li, X.-R., \& Liu, Y.: 2008c, Sci. China, Ser. G 51, $1633, \mathbf{Q}$

[266] Wasowicz, T. J.: 2009, Eur. Phys. J. D 53, 263, IS

[267] Wasowicz, T. J., Werbowy, S., Kwela, J., \& Drozdowski, R.: 2010, J. Opt. Soc. Am. B 27, 2628, IS

[268] Wei, H. G., Shi, J. R., Zhao, G., \& Liang, Z. T.: 2010, Astron. Astrophys. 522, p. A103, Q

[269] Wiese, W. L. \& Fuhr, J. R.: 2009, J. Phys. Chem. Ref. Data 38, 565, CP

[270] Witthoeft, M. C. \& Badnell, N. R.: 2008, Astron. Astrophys. 481, 543, QF

[271] Wolf, A. L., van den Berg, S. A., Gohle, C., Salumbides, E. J., Ubachs, W., \& Eikema, K. S. E.: 2008, Phys. Rev. A 78, 032511, WL, EL

[272] Wyart, J.-F. \& Lawler, J. E.: 2009a, Phys. Scr. 79, 045301, EL, CL, W, Q

[273] Wyart, J.-F., Tchang-Brillet, W.-U. L., Churilov, S. S., \& Ryabtsev, A. N.: 2008, Astron. Astrophys. 483, 339, Q

[274] Xie, J., Dai, C.-J., \& Li, M.: 2011, J. Phys. B 44, 015002, EL

[275] Xu, J.-X., Feng, Y.-Y., Sun, G.-J., \& Dai, Z.-W.: 2009, Chin. Phys. B 18, 3828, L

[276] y. Zhang, T. \& w. Zheng, N.: 2009, Chin. J. Chem. Phys. 22, 246, Q

[277] Yang, J.-H., Li, P., Zhang, J.-P., \& Li, H.-L.: 2008, Commun. Theor. Phys. 50, 468, Q

[278] Yang, Z. H., Du, S. B., Chang, H. W., Zhang, Y. P., Zhang, B. L., Xu, Q. M., Yu, D. Y., \& Cai, X. H.: 2010, J. Quant. Spectrosc. Radiat. Transfer 111, 2007, WL, CL

[279] Yang, Z. H., Du, S. B., Zeng, X. T., Chang, H. W., Zhang, B. L., Wang, W., Yu, D. Y., \& Cai, X. H.: 2009, Astron. J. 137, 4020, CL, WL

[280] Yildiz, M., Çelik, G., \& Kiliç, H. Ş.: 2009, Acta Phys. Pol. A 115, 641, Q

[281] Yoca, S. E., Biémont, E., Delahaye, F., Quinet, P., \& Zeippen, C. J.: 2008, Phys. Scr. 78, $025303, \mathbf{Q}$

[282] Young, P. R.: 2009, Astrophys. J. 691, L77, CL, WL, EL

[283] Young, P. R., Feldman, U., \& Lobel, A.: 2011, Astrophys. J., Suppl. Ser. 196, 23, CL, WL, EL

[284] Young, P. R. \& Landi, E.: 2009, Astrophys. J. 707, 173, CL, WL, EL

[285] Yüce, K., Castelli, F., \& Hubrig, S.: 2011a, Astron. Astrophys. 528, p. A37, CL, WL, EL, TE

[286] Zanna, G. D.: 2009a, Astron. Astrophys. 508, 501, CL, WL, EL, Q

[287] Zanna, G. D.: 2009c, Astron. Astrophys. 508, 513, CL, WL, EL, Q

[288] Zanna, G. D.: 2010a, Astron. Astrophys. 514, p. A41, EL, CL, WL, Q, QF

[289] Zanna, G. D. \& Ishikawa, Y.: 2009, Astron. Astrophys. 508, 1517, EL, CL, WL

[290] Zanna, G. D., Rozum, I., \& Badnell, N. R.: 2008, Astron. Astrophys. 487, 1203, Q, QF

[291] Zanna, G. D., Storey, P. J., \& Mason, H. E.: 2010a, Astron. Astrophys. 514, p. A40, EL, CL, WL, Q

[292] Zhang, T.-Y. \& Zheng, N.-W.: 2009, Acta Phys. Pol. A 116(2), 141, Q

[293] Zhang, T.-Y., Zheng, N.-W., \& Ma, D.-X.: 2009, Int. J. Quantum Chem. 109, 145, Q

[294] Zhang, W., Du, S., Feng, Y.-Y., Jiang, L.-Y., Jiang, Z.-K., \& Dai, Z.-W.: 2011a, Mon. Not. R. Astron. Soc. $413,1803, \mathbf{L}$

[295] Zhang, W., Feng, Y.-Y., \& Dai, Z.-W.: 2010a, J. Opt. Soc. Am. B 27, 2255, L

[296] Zhang, W., Feng, Y.-Y., Sun, G.-J., \& Dai, Z.-W.: 2010b, J. Phys. B 43, 235005, L

[297] Zhang, W., Feng, Y.-Y., Xu, J.-X., Palmeri, P., Quinet, P., Biémont, E., \& Dai, Z.-W.: 2010c, J. Phys. B 43, 205005, L 
[298] Zhang, W., Palmeri, P., Quinet, P., Biémont, E., Du, S., \& Dai, Z.-W.: 2010d, Phys. Rev. A $82,042507, \mathbf{E L}, \mathbf{C L}, \mathbf{L}$

[299] Zhang, Y., Xu, J.-X., Zhang, W., You, S., Ma, Z.-G., Han, L.-L., Li, P.-F., Sun, G.-J., Jiang, Z.-K., Yoca, S. E., Quinet, P., Biémont, E., \& Dai, Z.-W.: 2008a, Phys. Rev. A 78, $022505, \mathbf{L}$ 